\title{
The Impact of Skin Problems on the Quality of Life in Patients Treated with Anticancer Agents: A Cross-Sectional Study
}

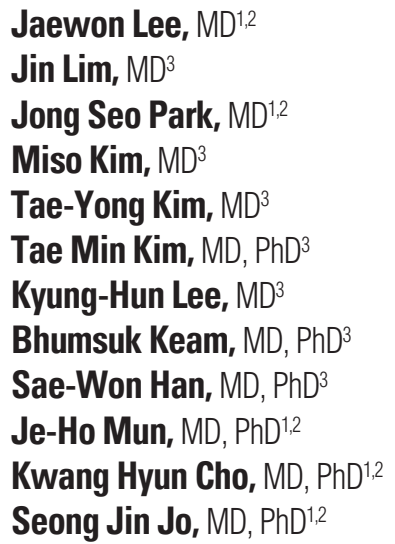

${ }^{1}$ Department of Dermatology, Seoul National University Hospital, Seoul, ${ }^{2}$ Center for Skin Cancer and Adverse Skin Reaction to Chemotherapeutics, Seoul National University Cancer Hospital, Seoul, ${ }^{3}$ Department of Internal Medicine, Seoul National University Hospital, Seoul, Korea

Correspondence: Seong Jin Jo, MD, PhD Department of Dermatology, Seoul National University Hospital and Center for Skin Cancer and Adverse Skin Reaction to Chemotherapeutics, Seoul National University Cancer Hospital, 101 Daehak-ro, Jongno-gu, Seoul 03080, Korea

Tel: 82-2-2072-4916

Fax: 82-2-742-7344

E-mail: sj.jo@snu.ac.kr

Received September 14, 2017

Accepted December 12, 2017

Published Online December 14, 2017

\begin{abstract}
Purpose
Patients treated with anticancer agents often experience a variety of treatment-related skin problems, which can impair their quality of life.

\section{Materials and Methods}

In this cross-sectional study, Dermatology Life Quality Index (DLQI) and clinical information were evaluated in patients under active anticancer treatment using a questionnaire survey and their medical records review.
\end{abstract}

Results

Of 375 evaluated subjects with anticancer therapy, 136 (36.27\%) and 114 (30.40\%) were treated for breast cancer and colorectal cancer, respectively. We found that women, breast cancer, targeted agent use, and longer duration of anticancer therapy were associated with higher dermatology-specific quality of life distraction. In addition, itching, dry skin, easy bruising, pigmentation, papulopustules on face, periungual inflammation, nail changes, and palmoplantar lesions were associated with significantly higher DLQI scores. Periungual inflammation and palmoplantar lesions scored the highest DLQI.

\section{Conclusion}

We believe our findings can be helpful to clinicians in counseling and managing the patients undergoing anticancer therapy.

\section{Introduction}

Patients with cancer undergoing treatment with anticancer agents often experience various skin problems, such as pruritus, dry skin, facial papulopustules, paronychia, etc. They

\author{
Key words \\ Adverse drug reaction, Antineoplastic agents, Quality of life, \\ Dermatology Life Quality Index
}

are at high risk of skin problems, because anticancer agents affect not only cancer cells, but also rapidly proliferating skin cells [1-4]. To date, significant progress has been made in the development of anticancer agents. A number of new anticancer agents, including targeted agents, have been developed and are widely used nowadays. Accordingly, new 
agent-related skin problems, such as facial papulopustules and hand-foot reaction induced by various tyrosine kinase inhibitors, also became prevalent [2-10].

Despite their high prevalence, the skin problems due to anticancer therapy are often neglected because clinicians and healthcare providers are usually more focused on clinical response of tumor itself or potentially life-threatening side effects such as neutropenia. However, adverse skin reactions to these therapies are sometimes so severe that they make significant disturbance to patients and the dose of anticancer agent should be adjusted at times, meaning that they can affect not only the patients' quality of life (QoL), but also optimal anticancer treatment. Therefore, they must not be ignored and should be evaluated thoroughly by managing physicians.

In this study, we aimed to evaluate the impact of anticancer agents on patients' QoL. The patients under active anticancer therapy were surveyed using the Dermatologic Life Quality Index (DLQI), a useful dermatology-specific health-related QoL questionnaire. DLQI score was analyzed according to various clinical factors, including demographics, anti-cancer therapy, and specific skin problems induced by anticancer agents.

\section{Materials and Methods}

\section{Study design}

We conducted a cross-sectional study using a questionnaire survey and their medical records review. Subjects suffering from cancer were recruited from the Seoul National University Cancer Hospital between February 2016 and April 2016. They were adult patients treated actively with anticancer agents at the time of the study; therefore, patients with only past history of anticancer therapy were excluded. Clinical information was obtained from both the review of medical records and questionnaires.

\section{Review of medical records}

The following clinical information was obtained for each subject from retrospective review of electronic medical records: (1) demographic data (sex, age); (2) type of cancer (cancer of the liver, thyroid, oral cavity, musculoskeletal, central nervous system, biliary ducts, colorectum, head and neck, bladder, kidney, stomach, breast, uterine cervix, prostate gland, pancreas, lung, skin, and hematologic malignancies); (3) type of anticancer agents: targeted agents (trastuzumab, cetuximab, imatinib, bevacizumab, erlotinib, gefitinib, sunitinib, crizotinib, sorafenib, rituximab, pertuzumab, and ramucirumab) and non-targeted chemotherapeutic agents (docetaxel, paclitaxel, cyclophosphamide, adriamycin, vincristine, 5-fluorouracil, cisplatin, oxaliplatin, carboplatin, etoposide, gemcitabine, capecitabine, irinotecan, navelbine, and pemetrexed); (4) the duration of current anticancer therapy; and (5) radiation therapy history.

\section{Contents of the questionnaire}

Using the questionnaire, subjects were asked if they underwent anticancer therapy at the time of the study and if they suffered from the following skin problems: (1) hair loss; (2) itching; (3) dry skin; (4) easy bruising; (5) pigmentation of lips and mucosae; (6) papulopustules on face, scalp, chest, and back; (7) periungual inflammation; (8) nail changes in color or shape; and (9) palmoplantar lesions with redness, exfoliation, and pain. The impact of skin problems on their QoL was evaluated using DLQI (Dermatology Life Quality Index, AY Finlay, GK Khan, April 1992; all rights reserved; License ID of this study: CUQoL1166), which includes questions about how much skin problems affect patients' QoL during a past week (symptoms like itching, prickling, or pain, shamefulness, disturbances in performing routine tasks, changes in the selection of clothes, impact on social activities or leisure, difficulties in physical, academic or occupational activities, relationship with other people, and sexual life, and distraction they had due to the treatment). A higher DLQI score means a greater impairment of QoL.

\section{Statistical analysis}

IBM SPSS statistics ver. 21.0 (IBM Corp., Armonk, NY) was used for statistical analysis. The differences of DLQI score associated with demographic factors (sex and age), the type of anticancer agents, radiation therapy history, and the type of skin problems were considered statistically significant if the $\mathrm{p}$-values $<0.05$ using Student's $t$ test. Jonckheere-Terpstra test was performed to find a correlation between the duration of anticancer therapy and DLQI scores.

\section{Ethical statement}

The study protocol was approved by the Institutional Review Board of Seoul National University Hospital (IRB No. 1601-058-734), and written informed consent was obtained from all subjects. 
Table 1. Clinical characteristics of the study population

\begin{tabular}{|c|c|c|c|c|}
\hline & \multirow{2}{*}{ Total } & \multicolumn{3}{|c|}{ Cancer type } \\
\hline & & Breast cancer & Colorectal cancer & Others \\
\hline No. of subjects & 375 & 136 & 114 & 125 \\
\hline \multicolumn{5}{|l|}{ Sex } \\
\hline Male & $155(41.3)$ & 0 & $63(55.3)$ & $92(73.6)$ \\
\hline Female & $220(58.7)$ & $136(100)$ & $51(44.7)$ & $33(26.4)$ \\
\hline \multicolumn{5}{|l|}{ Age (yr) } \\
\hline$<60$ & $223(59.5)$ & $119(87.5)$ & $54(47.4)$ & $50(40.0)$ \\
\hline$\geq 60$ & $152(40.5)$ & $17(12.5)$ & $60(52.6)$ & $75(60.0)$ \\
\hline \multicolumn{5}{|l|}{ Current chemotherapy } \\
\hline Non-targeted agents only & $267(71.2)$ & $103(75.7)$ & $74(64.9)$ & $90(72.0)$ \\
\hline Any targeted agents & $108(28.8)$ & $33(24.3)$ & $40(35.1)$ & $35(28.0)$ \\
\hline \multicolumn{5}{|l|}{ Duration of current chemotherapy (wk) } \\
\hline$\leq 12$ & $137(36.5)$ & $41(30.2)$ & $46(40.4)$ & $50(40.0)$ \\
\hline $13-24$ & $109(29.1)$ & $51(37.5)$ & $30(26.3)$ & $28(22.4)$ \\
\hline$\geq 25$ & $129(34.4)$ & $44(32.3)$ & $38(33.3)$ & $47(37.6)$ \\
\hline \multicolumn{5}{|l|}{ Radiotherapy history } \\
\hline Yes & $97(25.9)$ & $37(27.2)$ & $20(17.5)$ & $40(32.0)$ \\
\hline No & $278(74.1)$ & $99(72.8)$ & $94(82.5)$ & $85(68.0)$ \\
\hline \multicolumn{5}{|l|}{ Presence of skin problems } \\
\hline Hair loss & $283(75.5)$ & $125(91.9)$ & $77(67.5)$ & $81(64.8)$ \\
\hline Itching & $143(38.1)$ & $61(44.9)$ & $35(30.7)$ & $47(37.6)$ \\
\hline Dry skin & $230(61.4)$ & $99(72.8)$ & $66(57.9)$ & $65(52.0)$ \\
\hline Easy bruising & $113(30.1)$ & $49(36.0)$ & $31(27.2)$ & $33(26.4)$ \\
\hline Pigmentation of lips and oral mucosa & $108(28.8)$ & $37(27.2)$ & $45(39.5)$ & $26(20.8)$ \\
\hline Papulopustules on face, scalp, or trunk & $89(23.7)$ & $40(29.4)$ & $21(18.4)$ & $28(22.4)$ \\
\hline Periungual inflammation with pain & $88(23.5)$ & $42(30.9)$ & $21(18.4)$ & $25(20.0)$ \\
\hline Changes in nail color and shape & $196(52.3)$ & $102(75.0)$ & $49(43.0)$ & $45(36.0)$ \\
\hline Redness, exfoliation, or pain in palms and soles & $121(32.3)$ & $46(33.8)$ & $38(33.3)$ & $37(29.6)$ \\
\hline
\end{tabular}

Values are presented as number $(\%)$.

\section{Results}

\section{Demographic and clinical characteristics of study popu- lation}

A total of 380 patients visiting Seoul National University Cancer Hospital agreed to participate in this study. We excluded five subjects who were not treated with anticancer therapy at the time of the survey, and 375 subjects with anticancer therapy were finally enrolled, including $155(41.3 \%)$ men and $220(58.7 \%)$ women. The mean age was 56.35 years (range 25 to 84 years). The most patients had breast cancer $(136,36.3 \%)$, followed by patients with colorectal cancer $(n=114,30.4 \%)$, gastric cancer $(n=33,8.8 \%)$, lung cancer $(n=21,5.6 \%)$, and hematologic malignancy $(n=18,4.8 \%)$. Among them, 267 subjects $(71.2 \%)$ underwent treatment with non-targeted agents, while other 108 subjects $(28.8 \%)$ were on at least one targeted agent. We looked up specific types of chemotherapeutic agents used in two major cancer groups. First, among the patients with breast cancer, 71 patients were treated with cyclophosphamide, 65 were treated with doxorubicin, 51 with docetaxel, 24 with trastuzumab, 19 with paclitaxel, 11 with pertuzumab, nine with capecitabine, and eight with gemcitabine (counts are redundant because most of the patients were treated with more than one agent, e.g., combination regimen with doxorubicin and cyclophosphamide) Second, among the patients with colorectal cancer, 82 patients were treated with 5-fluorouracil, 65 with oxaliplatin, 34 with irinotecan, 28 with capecitabine, 27 with bevacizumab, and nine with cetuximab (counts are also redundant due to combination regimen such as 'FOLFOX'). Three hundred and fifty patients $(93.3 \%)$ complained at least more than one skin problem. All demographic and clinical variables and subgroup description of patients according to cancer type are summarized in Table 1. 
Table 2. Dermatologic Life Quality Index scores according to clinical characteristics

\begin{tabular}{|c|c|c|c|c|c|c|c|c|}
\hline & \multirow{2}{*}{\multicolumn{2}{|c|}{ Total }} & \multicolumn{6}{|c|}{ Cancer type } \\
\hline & & & \multicolumn{2}{|c|}{ Breast cancer } & \multicolumn{2}{|c|}{ Colorectal cancer } & \multicolumn{2}{|c|}{ Other cancer } \\
\hline & DLQI & p-value & DLQI & p-value & DLQI & p-value & DLQI & p-value \\
\hline \multicolumn{9}{|l|}{ Sex } \\
\hline Male & $2.94 \pm 0.36$ & 0.006 & & & $3.43 \pm 0.54$ & 0.840 & $2.61 \pm 1.49$ & 0.585 \\
\hline Female & $4.42 \pm 0.40$ & & $5.16 \pm 0.54$ & & $3.25 \pm 0.68$ & & $3.15 \pm 0.95$ & \\
\hline \multicolumn{9}{|l|}{ Age (yr) } \\
\hline$<60$ & $4.01 \pm 0.37$ & 0.374 & $5.13 \pm 0.57$ & 0.861 & $2.37 \pm 0.43$ & 0.024 & $3.14 \pm 0.77$ & 0.470 \\
\hline$\geq 60$ & $3.51 \pm 0.42$ & & $5.41 \pm 1.56$ & & $4.23 \pm 0.69$ & & $2.49 \pm 0.52$ & \\
\hline \multicolumn{9}{|l|}{ Current chemotherapy } \\
\hline Non-targeted agents only & $3.45 \pm 0.33$ & 0.046 & $4.72 \pm 0.61$ & 0.145 & $2.86 \pm 0.53$ & 0.120 & $2.49 \pm 0.49$ & 0.335 \\
\hline Any targeted agents & $4.69 \pm 0.54$ & & $6.55 \pm 1.14$ & & $4.25 \pm 0.70$ & & $3.43 \pm 0.92$ & \\
\hline \multicolumn{9}{|l|}{$\begin{array}{l}\text { Duration of current } \\
\text { chemotherapy }(w k)^{a)}\end{array}$} \\
\hline$\leq 12$ & $3.01 \pm 0.42$ & & $3.54 \pm 0.77$ & & $2.54 \pm 0.61$ & & $3.02 \pm 0.78$ & \\
\hline $13-24$ & $4.15 \pm 0.52$ & & $4.71 \pm 0.75$ & & $3.67 \pm 0.94$ & & $3.64 \pm 1.10$ & \\
\hline$\geq 25$ & $4.36 \pm 0.52$ & 0.019 & $7.20 \pm 1.17$ & 0.010 & $4.08 \pm 0.73$ & 0.066 & $1.94 \pm 0.47$ & 0.863 \\
\hline \multicolumn{9}{|l|}{ Radiotherapy history } \\
\hline Yes & $4.74 \pm 0.71$ & 0.100 & $7.95 \pm 1.36$ & 0.011 & $2.50 \pm 1.11$ & 0.357 & $2.90 \pm 0.83$ & 0.817 \\
\hline No & $3.48 \pm 0.28$ & & $4.12 \pm 0.50$ & & $3.53 \pm 0.46$ & & $2.68 \pm 0.51$ & \\
\hline
\end{tabular}

Values are presented as mean \pm standard error. DLQI, Dermatologic Life Quality Index. ${ }^{a}$ Jonckheere-Terpstra test was performed to find the correlation between the duration of anticancer therapy and DLQI scores. Student's t-test was performed for other variables.

\section{Assessment of DLQI scores according to demographic and clinical factors}

Mean DLQI score of total study population is $3.74 \pm 0.27$ (mean \pm standard error). DLQI scores according to various clinical factors are presented in Table 2 . Female subjects presented higher DLQI scores (mean \pm standard error, 4.42 \pm 0.40 ) than males (2.94 \pm 0.36$)$. Patients on targeted therapies had significantly higher DLQI scores $(4.69 \pm 0.54)$ than patients on non-targeted therapies ( $3.45 \pm 0.33)$. In addition, JonckheereTerpstra test revealed more distraction if anticancer treatment was administered for a longer period $(\mathrm{J}-\mathrm{T}$ value $=+2.35$; $\mathrm{p}=0.019$ ). However, DLQI score was not significantly associated with age or the history of radiation therapy. There were differences in DLQI scores between patients with different types of cancer. The patients with breast cancer complained more of skin-related distraction in their daily life (5.16 \pm 0.54$)$ than those with colorectal $(3.35 \pm 0.42)$ and gastric cancers (2.70 \pm 0.87$)$, as shown in Fig. 1 .

We did additional sub-analysis according to the specific questions of DLQI. Among the 10 questions of DLQI, breast cancer patients reported higher scores with statistical significance than the other types of cancer in 9 out of 10 questions. Question number 7 ('Over the last week, has your skin pre- vented you from working or studying?') was the only one with no significant difference between the type of cancer (data not shown).

In a subgroup analysis according to the type of cancer, except for patients with breast cancer, sex differences in QoL were not observed in patients with different types of cancer. In colorectal cancer group, patients aged 60 years or older had more distraction than those aged younger than 60 years. Moreover, patients with a history of radiation therapy in breast cancer groups were found to have more dermatologyrelated distraction in daily life (Table 2).

\section{Assessment of DLQI scores according to the skin prob- lems induced by anticancer therapy}

Hair loss was the most frequently reported problem, followed by dry skin and changes in nail color or shape (Table 1). We found similar patterns in the subgroup analysis by cancer types. We compared the DLQI scores between the subjects with and without a specific skin problem and found that all investigated skin problems, except hair loss, were associated with significantly higher DLQI scores (Fig. 2). The patients with periungual inflammation presented the highest DLQI score $(7.43 \pm 0.65)$, followed by those with palmoplantar 


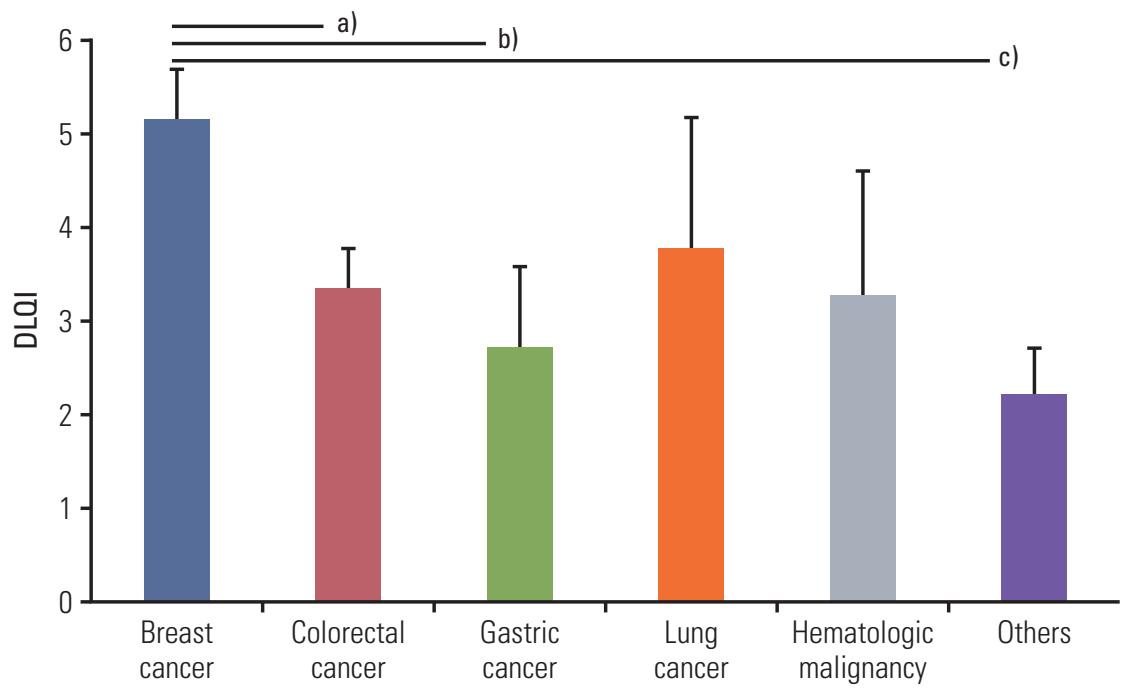

Fig. 1. Comparison of mean DLQI scores in patients between the type of cancer. Data are mean \pm stdandard error bars using the Independent T-test. Patients with breast cancer have more distraction in their life than those with any other type of cancer. ${ }^{a}$ Versus colorectal cancer; 5.16 vs. $3.35, p=0.038$, b) Versus gastric cancer; 5.16 vs. $2.70, p=0.034$, ${ }^{c}$ Versus others; 5.16 vs. 2.11, $\mathrm{p}=0.005$.

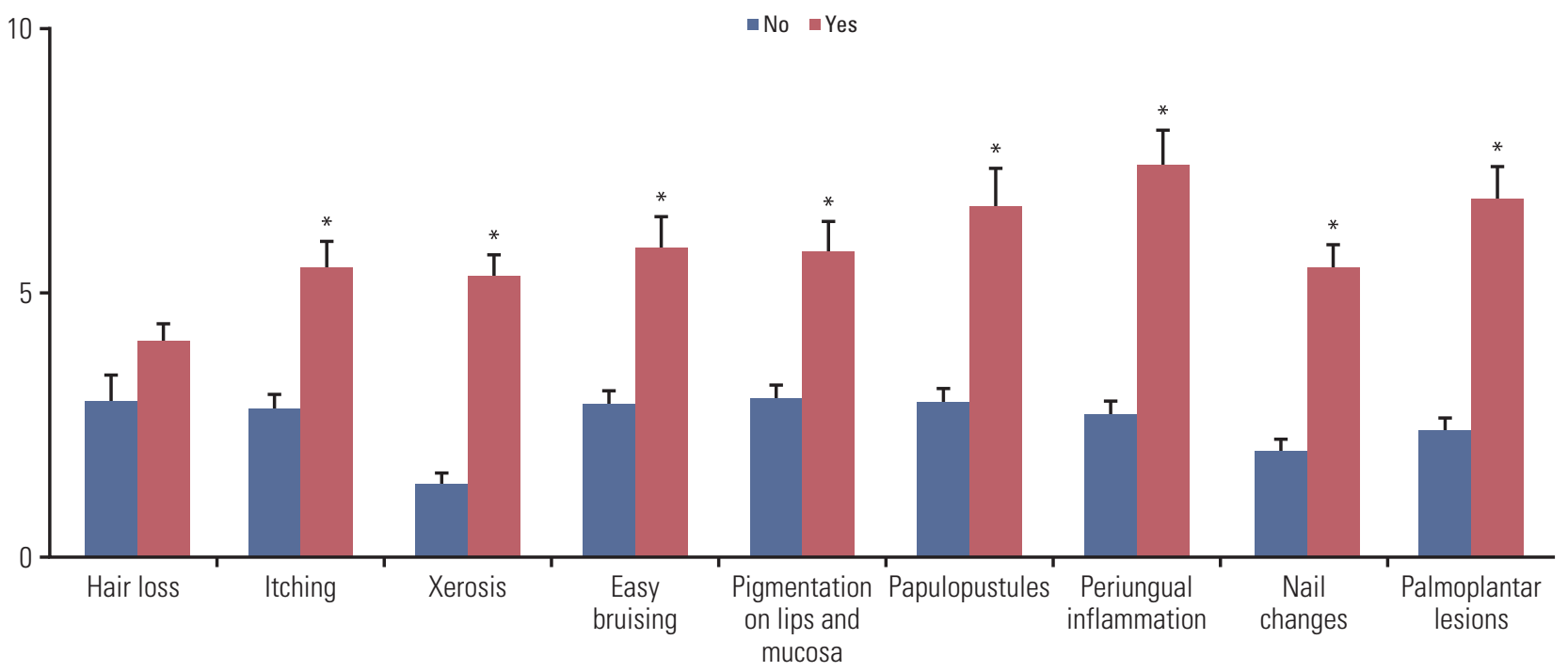

Fig. 2. Differences in mean Dermatology Life Quality Index when the patients have a certain specific skin problems or not. *Statistically significant difference.

lesions (6.78 \pm 0.61$)$, and papulopustules (6.64 \pm 0.72$)$. Similar patterns were also found in the subgroup analysis by the type of diagnosed cancer (S1 Table).

\section{Discussion}

Despite the growing interest and attention on anticancer therapy-induced skin problems, their impact on QoL distraction was not much considered. In particular, clinical factors 
that cause more distraction in dermatology-related QoL have not been studied, although this information can help clinicians to counsel patients and manage their skin problems during anticancer therapy [11-15]. This study demonstrated that dermatology-related QoL of the patients undergoing anticancer therapy was more distracted in women, in patients with longer duration of anticancer therapy, and in those with breast cancer.

In this study, we used DLQI to evaluate the dermatologyrelated QoL during anticancer therapy. What clinicians see is sometimes in disagreement with what patients actually feel, especially in terms of the impact on QoL $[1,11,16]$. Therefore, patient-assessed tools that show the effect of the problem on their QoL are useful in the evaluation. DLQI is the first dermatology-specific health-related QoL questionnaire, and its validity and reliability have been tested for over 20 years in various skin diseases. It is a simple, practical, patient-assessed questionnaire technique evaluating the impact of many skin diseases and their treatment on patients' QoL [17-20].

Several studies reported that older patients with skin disorders presented higher DLQI scores than younger ones $[21,22]$. However, unlike our first expectations, age did not affect dermatology-specific QoL of the patients in this study, except for the patients with colorectal cancer. This finding suggests that the effect of anticancer therapy on the skin is not much affected with age.

Instead, women presented higher DLQI than men in this study. It is frequently seen that women report higher rates of morbidity and distraction in many diseases $[23,24]$. This fact can be explained by the influence of sex-specific differences on patients' perception of symptoms. According to Holm et al. [25], self-reported morbidity is more consistent with the disease severity among women than men with atopic dermatitis. This means that men are less likely to complain of their symptoms although their condition is more severe than in women.

However, considering that the subjects with breast cancer presented higher DLQI and that the difference of DLQI between men and women was not found in colorectal and other cancers, the higher DLQI score in women found in this study might be related to the fact that more than half of the women were patients with breast cancer. Although patients with breast cancer were significantly younger than other patients, a subgroup analysis in them suggested that the age was not a causative factor of high DLQI score. Unlike other patients with cancer, patients with breast cancer showed a significant difference in the DLQI score between those with and without radiotherapy history.

Another hypothesis explaining higher DLQI scores in breast cancer group in this study is that the differences in the type of chemotherapeutic agents between cancer groups may have had an effect on the difference in DLQI scores. In breast cancer group, the majority of patients were treated with taxanes (docetaxel, paclitaxel) or anthracyclines (doxorubicin), which was a unique pattern comparing with other cancer groups. They have been well known to induce nail and periungual problems $[26,27]$, that can explain higher DLQI scores in breast cancer because the patients with periungual inflammation presented higher DLQI scores than ones with any other skin problems. Even though direct comparison is impossible because most of the patients were treated with multiple agent regimen so that the effect of the agents may have been mixed, it can be one good hypothesis for that.

Recently, targeted agents, including epidermal growth factor receptor inhibitors became widely used, and some investigators studied their impact on dermatology-specific QoL. Rosen et al. [14] previously found more distractions in QoL in patients treated with targeted therapy than in patients with non-targeted therapy with regards to total Skindex-16 scores evaluated in 283 patients with cancer. On the contrary, Unger et al. [12] showed no significant difference in QoL between targeted-agents-only group and patients who was treated with both targeted and classical chemotherapeutic agents. The discrepancy may result from the differences in study designs. Rosen et al. [14] enrolled patients in a dermatology referral clinic; therefore, there is a possibility that more severe cases were included in the study. Unger et al. [12] compared patients with colorectal cancer treated with cetuximab only and those treated with a combination of cetuximab and nontargeted agents. In our study, we included patients who underwent anticancer therapy regardless of referral to dermatologic office, in order to represent better the population composed of the patients with various degree of severity in skin problems. In concordance with Rosen et al. [14], we found that patients on targeted therapy had significantly more distraction in QoL than those on non-targeted therapy. Additionally, we found that the top three skin problems with the highest DLQI scores were periungual inflammation and papulopustules, which are frequently observed in patients treated with targeted agents [7-11,28]. Further studies are needed to confirm this finding.

In the comparison between the patients with and without a specific skin problem, we found no significant difference between the patients with and without hair loss induced by anticancer therapy. Although hair loss has been well-known to negatively impact the QoL in patients with alopecia areata [29] and patterned hair loss [30,31], our study suggested that hair loss induced by anticancer therapy did not cause additional distress in dermatology-specific QoL. Perhaps the disfiguration itself had little effect on the QoL distraction in patients treated with anticancer therapy if it was not associated with discomfort, such as itching or pain. In addition, patients expected the development of hair loss during anti- 
cancer therapy and took it for granted, whereas they did not expect other skin problems to be induced by anticancer therapy because hair loss is one of the best-known adverse reactions of anticancer therapy.

Interestingly, periungual inflammation and palmoplantar lesion were the top two skin problems with the highest DLQI. They are usually accompanied by pain, and limitation of instrumental and self-care daily activities which makes them distinguishable from other problems [32,33]. These findings suggest that pain is the most important factor that has negative effect on patients' QoL.

The limitation of this study is that there is a disproportion of clinical characteristics of the study population. Breast cancer and colorectal cancer together affected over $60 \%$ of the patients. This may have caused the disproportion of other factors, such as sex and anticancer therapy regimens consequently.

In conclusion, this study showed that women, breast can- cer, targeted agent use, and longer duration of anticancer therapy were associated with higher dermatology-specific QoL distraction. All investigated skin problems, except hair loss, significantly impacted dermatology-specific QoL, and periungual inflammation and palmoplantar lesions scored the highest DLQI. Our findings can be helpful to clinicians in counseling and managing the patients undergoing anticancer therapy.

\section{Electronic Supplementary Material}

Supplementary materials are available at Cancer Research and Treatment website (http:// www.e-crt.org).

\section{Conflicts of Interest}

Conflict of interest relevant to this article was not reported.

\section{References}

1. Byun HJ, Lee HJ, Yang JI, Kim KH, Park KO, Park SM, et al. Daily skin care habits and the risk of skin eruptions and symptoms in cancer patients. Ann Oncol. 2012;23:1992-8.

2. Boone SL, Rademaker A, Liu D, Pfeiffer C, Mauro DJ, Lacouture ME. Impact and management of skin toxicity associated with anti-epidermal growth factor receptor therapy: survey results. Oncology. 2007;72:152-9.

3. Lacouture ME. The growing importance of skin toxicity in EGFR inhibitor therapy. Oncology (Williston Park). 2009;23: $194,196$.

4. Huang V, Anadkat M. Dermatologic manifestations of cytotoxic therapy. Dermatol Ther. 2011;24:401-10.

5. Jatoi A, Rowland K, Sloan JA, Gross HM, Fishkin PA, Kahanic $\mathrm{SP}$, et al. Tetracycline to prevent epidermal growth factor receptor inhibitor-induced skin rashes: results of a placebocontrolled trial from the North Central Cancer Treatment Group (N03CB). Cancer. 2008;113:847-53.

6. Garden BC, Wu S, Lacouture ME. The risk of nail changes with epidermal growth factor receptor inhibitors: a systematic review of the literature and meta-analysis. J Am Acad Dermatol. 2012;67:400-8.

7. Balagula Y, Lacouture ME, Cotliar JA. Dermatologic toxicities of targeted anticancer therapies. J Support Oncol. 2010;8: 149-61.

8. Robert C, Soria JC, Spatz A, Le Cesne A, Malka D, Pautier P, et al. Cutaneous side-effects of kinase inhibitors and blocking antibodies. Lancet Oncol. 2005;6:491-500.

9. Osio A, Mateus C, Soria JC, Massard C, Malka D, Boige V, et al. Cutaneous side-effects in patients on long-term treatment with epidermal growth factor receptor inhibitors. Br J Derma- tol. 2009;161:515-21.

10. Nardone B, Hensley JR, Kulik L, West DP, Mulcahy M, Rademaker A, et al. The effect of hand-foot skin reaction associated with the multikinase inhibitors sorafenib and sunitinib on health-related quality of life. J Drugs Dermatol. 2012;11:e61-5.

11. Sibaud V, Dalenc F, Chevreau C, Roche H, Delord JP, Mourey L, et al. HFS-14, a specific quality of life scale developed for patients suffering from hand-foot syndrome. Oncologist. 2011;16:1469-78.

12. Unger K, Niehammer U, Hahn A, Goerdt S, Schumann M, Thum S, et al. Treatment of metastatic colorectal cancer with cetuximab: influence on the quality of life. Z Gastroenterol. 2013;51:733-9.

13. Chan A, Cameron MC, Garden B, Boers-Doets CB, Schindler $\mathrm{K}$, Epstein JB, et al. A systematic review of patient-reported outcome instruments of dermatologic adverse events associated with targeted cancer therapies. Support Care Cancer. 2015;23:2231-44.

14. Rosen AC, Case EC, Dusza SW, Balagula Y, Gordon J, West DP, et al. Impact of dermatologic adverse events on quality of life in 283 cancer patients: a questionnaire study in a dermatology referral clinic. Am J Clin Dermatol. 2013;14:327-33.

15. Charles C, Bungener C, Razavi D, Mateus C, Routier E, Lanoy E, et al. Impact of dermatologic adverse events induced by targeted therapies on quality of life. Crit Rev Oncol Hematol. 2016;101:158-68.

16. Basch E, Iasonos A, McDonough T, Barz A, Culkin A, Kris $\mathrm{MG}$, et al. Patient versus clinician symptom reporting using the National Cancer Institute Common Terminology Criteria for Adverse Events: results of a questionnaire-based study. 
Lancet Oncol. 2006;7:903-9.

17. Finlay AY, Khan GK. Dermatology Life Quality Index (DLQI): a simple practical measure for routine clinical use. Clin Exp Dermatol. 1994;19:210-6.

18. Lewis V, Finlay AY. 10 years experience of the Dermatology Life Quality Index (DLQI). J Investig Dermatol Symp Proc. 2004;9:169-80.

19. Finlay AY, Basra MK, Piguet V, Salek MS. Dermatology life quality index (DLQI): a paradigm shift to patient-centered outcomes. J Invest Dermatol. 2012;132:2464-5.

20. Basra MK, Salek MS, Camilleri L, Sturkey R, Finlay AY. Determining the minimal clinically important difference and responsiveness of the Dermatology Life Quality Index (DLQI): further data. Dermatology. 2015;230:27-33.

21. Sampogna F, Chren MM, Melchi CF, Pasquini P, Tabolli S, Abeni D, et al. Age, gender, quality of life and psychological distress in patients hospitalized with psoriasis. Br J Dermatol. 2006;154:325-31.

22. Shah M, Coates M. An assessment of the quality of life in older patients with skin disease. Br J Dermatol. 2006;154:150-3.

23. Nakao M, Fricchione G, Zuttermeister PC, Myers P, Barsky AJ, Benson H. Effects of gender and marital status on somatic symptoms of patients attending a mind/body medicine clinic. Behav Med. 2001;26:159-68.

24. Macintyre S, Ford G, Hunt K. Do women 'over-report' morbidity? Men's and women's responses to structured prompting on a standard question on long standing illness. Soc Sci Med. 1999;48:89-98.
25. Holm EA, Esmann S, Jemec GB. Does visible atopic dermatitis affect quality of life more in women than in men? Gend Med. 2004;1:125-30.

26. Gilbar P, Hain A, Peereboom VM. Nail toxicity induced by cancer chemotherapy. J Oncol Pharm Pract. 2009;15:143-55.

27. Minisini AM, Tosti A, Sobrero AF, Mansutti M, Piraccini BM, Sacco C, et al. Taxane-induced nail changes: incidence, clinical presentation and outcome. Ann Oncol. 2003;14:333-7.

28. Joshi SS, Ortiz S, Witherspoon JN, Rademaker A, West DP, Anderson R, et al. Effects of epidermal growth factor receptor inhibitor-induced dermatologic toxicities on quality of life. Cancer. 2010;116:3916-23.

29. Gupta MA, Gupta AK. Depression and suicidal ideation in dermatology patients with acne, alopecia areata, atopic dermatitis and psoriasis. Br J Dermatol. 1998;139:846-50.

30. Cash TF. The psychological effects of androgenetic alopecia in men. J Am Acad Dermatol. 1992;26:926-31.

31. Cash TF. The psychology of hair loss and its implications for patient care. Clin Dermatol. 2001;19:161-6.

32. Ljosaa TM, Mork C, Stubhaug A, Moum T, Wahl AK. Skin pain and skin discomfort is associated with quality of life in patients with psoriasis. J Eur Acad Dermatol Venereol. 2012;26:29-35.

33. Mendez-Flores S, Orozco-Topete R, Bermudez-Bermejo P, Hernandez-Molina G. Pain and pruritus in cutaneous lupus: their association with dermatologic quality of life and disease activity. Clin Exp Rheumatol. 2013;31:940-2. 